4. One point of intersection, and contact of second order at a second point;

5. Contact of third order.

Proper configurations exist for types 1 and 4, and do not exist for types 2, 3, and 5. For a given pencil of six conics there are four configurations for type 1, but only one for type 4 .

For the one-dimensional case, we have the configuration $\Gamma_{5,1}^{3}$. on a line, and the pencil of quadrics is simply five pairs of points in involution.* We have two types, viz., the fixed points of the involution are distinct or they are coincident. Proper configurations exist for both cases. Given the five point pairs, there are two configurations determined in the first case, but only one in the second. This one-dimensional case is readily seen if the whole figure is projected upon a conic.

\title{
ON THE USE OF $n$-FOLD RIEMANN SPACES IN APPLIED MATHEMATICS.
}

BY PROFESSOR JAMES MCMAHON.

THE object of this article is to show that the conception of a Riemann surface has important physical bearings, and to indicate in a general way what kind of physical problems have been solved or may be solvable by the use of such $n$-fold surfaces or analogous manifold regions in three dimensions. The most recent work in this line constitutes the highest point yet reached in the application of modern function theory to physical problems. It is very noteworthy that a theory which was developed by following out purely intellectual relations, without any reference to the world of sense, should afterwards find unexpected applications and correspondences in the physical universe.

The conceptions of multiform functions, and of multiple spaces in which such functions are made uniform, furnish elegant solutions of some important problems in the theories of potential, electricity, light, sound, heat, and fluid motion. To give greater clearness to what follows, it may be well to take a simple illustration of a three-valued potential function in two dimensions, and show how to make it one-valued on a threefold Riemann surface. Let $(\rho, \theta)$ be the polar coordinates of a

\footnotetext{
*Author's paper, loc. cit., p. 5, footnote.
} 
point in a plane, and consider the function $\rho^{\frac{1}{3}} \cos \frac{1}{3} \theta$, which satisfies Laplace's equation, and has in general three real and distinct values at the point $(\rho, \theta)$, the second and third values being obtained by changing $\theta$ into $\theta+2 \pi$ and $\theta+4 \pi$ in succession. On making successive circuits around the origin, the first value changes continuously into the second, the second into the third, and the third into the first. It will be seen that a pair of values are equal when $\theta=2 \pi$, another pair when $\theta=4 \pi$, and the other pair when $\theta=6 \pi$. Now let us imagine the plane covered over by two other plane sheets in such a way that a revolving radius vector shall trace out the first sheet from $\theta=0$ to $\theta=2 \pi$, and then by continuous motion trace out the second sheet from $\theta=2 \pi$ to $\theta=4 \pi$, and the third sheet from $\theta=4 \pi$ to $\theta=6 \pi$, passing then into the first sheet again, and so on. The three pairs of coordinates $(\rho, \theta),(\rho, \theta+2 \pi),(\rho$, $\theta+4 \pi)$, which before represented coincident points, are now regarded as representing three underlying points in separate sheets, and these points are mutually accessible only by a circuit around the origin (or winding point). The positive half of the initial line, being the threshold for passage from one sheet to another, is called the common branch line. The given function is now single-valued and continuous over the whole three-sheeted Riemann surface.

In an analogous way, the same function in cylindrical coordinates could be uniformized by taking the axis of $z$ as a winding line, and the initial half plane as a branch membrane, giving rise to a three-fold Riemann space, which fills the whole of our space three times.

The first concrete example that I can find of a multiform potential in three dimensions is contained in a letter from Professor P. Appell to Professor F. Klein, published in the Mathematische Annalen, 1887, a few months before Klein delivered his lectures on potential theory (summer 1888). Appell cites a two-valued function that satisfies Laplace's three-dimensional equation, and has a certain circle for a singular line, such that the two values of the function interchange when a circuit is made around this winding line. He says nothing, however, about uniformizing the function by introducing another copy (or fold) of three-dimensional space, and using the singular (or winding line) as the boundary of a branch membrane (or door) from one fold into the other. In 1891 Dr. Pockels, in his treatise on the solutions of the "wave potential" equation for 
various boundary conditions, pointed out the desirability, from both a mathematical and physical standpoint, of studying the multiform solutions of this equation, and the mode of uniformizing them on Riemann surfaces, or analogous threedimensional spaces. He suggested that the Green functions for certain boundaries in such spaces might correspond to some unsolved physical problems. It is not unlikely that this idea may have been derived from Klein's lectures on potential theory (1888), of which Pockels made free use, but which I have not yet been able to consult directly, as they have been preserved only in manuscript.

Apparently the first application of the notion of a multiple space to a concrete physical problem was made by Professor A. Sommerfeld in articles in the Mathematische Annalen, 1894 and 1896, on heat conduction and diffraction, and in another article in the following year in the Proceedings of the London Mathematical Society, on "Multiform potentials in space." In these articles Sommerfeld shows among other things that certain boundary problems, in which the Kelvin image method fails to furnish the true Green function, on account of the existence of more than one pole within the given boundary, can be solved by the conception of the Riemann space such that each fold shall contain only one of the poles (or images). Moreover, he proves, by function theory methods, an important fundamental principle, which has been called Sommerfeld's theorem, namely, that Green's general theorem is applicable without modification to functions that have been uniformized in a Riemann space, provided the winding lines are excluded from the region of integration by means of thin tubes. From this he easily proves the uniqueness of the solution of Dirichlet's problem for any assigned boundary in the multiple space.

Sommerfeld first applies his generalized image method to find Green's function for a wedge-shaped region bounded by two infinite half planes and the infinite sphere, the angle of the planes being $n \pi / m$. Starting with the assigned pole, and reflecting successively with regard to the two planes until the series of images closes up, we find that there are $n$ poles within the given boundary; hence we use an $n$-fold Riemann space having a pole in each fold, the straight edge being the winding line. The appropriate coordinate system is cylindrical, having the winding line as axis. By introducing into the elementary Green function a complex parameter, and then performing a 
contour integration, using Cauchy's method of residues, a gener-. alized function is obtained for each of the poles, and these are combined in such a way as to realize the conditions for the required Green's function in the assigned physical region. By a similar method the closely related problem of the diffraction of waves of light or electricity in passing through a wedge shaped medium has been solved by H. S. Carslaw (Philosophical Magazine, 1903).

The case in which $n=2$ and $m=1$ solves various potential and diffraction problems for the infinite half plane. In particular, the cases of waves issuing from a line source or a point source against the half-plane have been fully worked out by Sommerfeld, Carslaw and others. If the screen extended to infinity both ways, the usual method of images could easily be applied, as the image of the source would not be situated in the given region, and the action of the screen could be perfectly imitated by a combination of the source and its image, the screen being removed. As this is evidently not feasible in the case of the half-plane, we have to place the image in a second fold of a Riemann space, and find the proper Green function as already indicated. It is to be observed that the effects of the two sources have to be combined in an appropriate way according as the screen is perfectly reflecting or perfectly absorbing, so as to fulfill the proper conditions at the surface of the screen, which is then thought of as replaced by a branch membrane (or open door) between the two folds of the Riemann space, one of the folds being the ordinary physical space itself, and the other a copy coincident with it, but supposed capable of sustaining a wave motion of its own independent of the motion at underlying points in the other fold, and only influenced by the energy which enters and leaves by the open door.

In this connection it may be of interest to mention a criticism made by Professor K. Schwarzschild (Mathematische Annalen, 1901). Alluding to the case in which the screen is perfectly absorbing he remarks that while the second fold of the Riemann space does seem at first sight to carry away the motion so as to realize the proper conditions at the absorbing screen, yet there is nothing to prevent the latter fold from delivering some of this motion back into the first fold (or natural space) after another circuit around the winding line, thus disturbing the adjustment. Sommerfeld skillfully overcomes this objection (Zeitschrift, 1901, page 36) by substituting for the two-fold 
space an $n$-fold one, and after forming the proper function, letting $n$ become infinite ; the limit so obtained must give a correct result, as it would take an infinite time for the energy to wind back into the first fold. This is equivalent to its passing out of the universe, and thus the perfectly absorbing screen is exactly imitated. The case of the perfectly reflecting screen is not open to any similar objection. Moreover, no objection can be raised against the general method on account of any supposed physical difficulty in the coexistence of different motions in the different folds, for, even if that be conceded, there remains a firm analytical ground for the method, in the generalized Green theorem and Dirichlet principle; and in any case it is not difficult to verify that the function finally obtained satisfies all the prescribed conditions in the assigned physical space.

In the article on multiform potentials already quoted, Sommerfeld next considered the more difficult case of an infinite plane out of which is cut an infinite strip bounded by two parallel lines. The suitable Riemann space has two winding lines, and the appropriate coordinate system is the bipolar system used so skillfully by Maxwell. In a later article (Zeitschrift, 1901, page 71) on "the bending of Roentgen rays," Sommerfeld points out that the function found in his previous article does not satisfy all the conditions, and that he has not yet found the true Green function for the region in question, although it is certain that one exists. It may be of interest to state, however, that this "problem of the slit" has been solved by an ingenious method of successive approximation by Schwarzschild in the paper already quoted (Mathematische Annalen,1901). He applies Green's function for the infinite half-plane to each of the two given half-planes, in endless succession, the boundary conditions for each application being selected so as to neutralize the error left by the preceding one; this in turn disturbs the adjustment on the other half-plane, but it is proved that the successive errors diminish rapidly, and that the series is convergent.

The article contributed by Sommerfeld to the London Mathematical Society gave rise to a series of further developments along similar lines by British mathematicians and physicists. The following important papers may be mentioned:

"On multiform solutions of the differential equations of physical mathematics" (Proceedings of the London Mathenıatical Society, volume 30 (1898)) by H. S. Carslaw. 
"On electric current sheets" ( Proceedings of the London Mathematical Society, volume 31 (1899)) by J. H. Jeans.

"On the determination of Green's function by means of cylindrical and spherical harmonics" (Proceedings of the Edinburgh Mathematical Society, 1900) by J. Dougal.

"The Green function for a circular disk with applications to electrostatics" (Cambridge Philosophical Transactions, volume $18(1900))$ by E. W. Hobson.

"The use of contour integration in the problem of diffraction by a wedge of any angle" (Philosophical Magazine, 1903) by $\mathrm{H}$. S. Carslaw.

"On many-valued Newtonian potentials" (Proceedings of the London Mathematical Society, 1904) by A. C. Dixon.

"On Sommerfeld's diffraction problem" (Proceedings of the London Mathematical Society, 1907) by Horace Lamb.

The curvilinear system used by Hobson in dealing with the circular disk is especially noteworthy. It is the peripolar system introduced by C. Neumann in treating of anchor ring problems. The three families of orthogonal surfaces which correspond to these coordinates consist (1) of a system of spherical bowls with the boundary of the disk as a common rim, and including the disk itself as a limiting member, (2) a system of anchor rings with the rim as a limiting member, and (3) a system of planes passing through the axis of the disk. The appropriate Riemann surface is two-sheeted, having the rim for the winding line and the disk for a branch membrane.

The next problem to be attacked by this method would perhaps be the diffraction of plane waves by an infinite plane screen perforated by a circular aperture; and the same coordinate system would naturally be tried. The elliptic aperture would be much more difficult. Then one might try a case of the still unsolved problem of discontinuous fluid motion in three dimensions, such as the following: A large mass of water (or other incompressible fluid) is moving with uniform velocity in one direction, and is partly obstructed by a fixed circular disk placed perpendicularly to the line of motion; to find the system of stream lines, especially those which pass around the edge and form a surface of discontinuity bounding the region of still water.

A similar problem may also be stated for a circular aperture in a fixed plane; and in both problems water may be replaced by air. It would seem that the idea of a Riemann surface (or 
space) is well fitted to bridge over a discontinuity, by the introduction of an extra copy of space in which the discontinuity is filled up in some suitable way.

The success, however partial, which has attended the quest for mathematical functions that express more and more complicated physical relationships, tends to strengthen our faith in some deep-lying correspondence between the world of nature and the world of the pure intellect. It remains for mathematicians and physicists to discover new winding lines and open doors.

\section{MATHEMATICAL APPOINTMENTS IN COLLEGES AND UNIVERSITIES.}

BY PROFESSOR E. J. WILCZYYNSKI.

AT the meeting of the Chicago Section held January 2, 1909, a committee was appointed for the purpose of investigating the possibility of improving the character of mathematical appointments in our colleges and universities. I submitted for the consideration of the members of the committee an adaption of the Italian system which appeared to me to be applicable to American conditions. My views were not shared by the other members of the committee, and our official report has been incorporated in the secretary's report of the April meeting of the Chicago Section.

The suggestions which I offered to the committee may be arranged under three heads.

First. Publication of vacancies. - There should be established in the Bulletin a special department devoted to the announcement of vacancies. The administrative heads of all colleges and universities should be informed of the existence of this department and should be requested to avail themselves of it. The announcements should be as explicit as possible on the items of title, salary, grade and amount of work, probable date of appointment, address to which applications should be sent, date after which no applications will be considered, whether a specialist is desired or preferred, and if so in what special subject. If any of these items are subject to any uncertainty - if, for instance, as is often the case, an institution is not willing to announce a definite sum as salary because it would be willing to pay widely different salaries according to 